\title{
Managing women presenting with urinary incontinence: is hardiness significant?
}

\author{
Fidan Israfil-Bayli $^{1,3} \cdot$ Sheila Lowe $^{1}$ - Laura Spurgeon ${ }^{2}$. \\ Philip Toozs-Hobson ${ }^{1}$
}

Received: 29 December 2014 / Accepted: 5 May 2015 /Published online: 20 May 2015

(C) The International Urogynecological Association 2015

\section{Introduction}

Urinary incontinence (UI) in women constitutes a very significant problem for the individual, her family and health care services. UI (including overactive bladder, stress, urge and mixed incontinence) is thought to affect around 9.6 million women in the UK [1] and is a reported symptom of up to $46 \%$ of patients attending primary care clinics [2]. However, as precise prevalence estimates are difficult to ascertain because of under-reporting, it is conceivable that the actual number of people with UI is considerably higher [2]. This shortfall in presentation is thought to be due to a range of reasons such as symptom severity, embarrassment, lack of information and/ or confidence regarding treatment options [1]. Conservative estimates of the cost to the National Health Service (NHS) of managing UI suggest that annual expenditure in 2001 was in excess of $£ 350$ million [3], the majority spent on nonpharmacological interventions, i.e. surgery and secondary care [4]. The impact of UI on quality of life is well-documented, affecting as it does personal relationships, social activities, work, and emotional and psychological well-being [5]. Because of the scope and severity of the problems experienced by many of these women, it might be expected that they would be more inclined to present for treatment than is currently the

Fidan Israfil-Bayli

f.israfilbayli@nhs.net

1 Birmingham Women's Hospital NHS Foundation Trust, Mindelsohn Way, Edgbaston, Birmingham B15 2TG, UK

2 Medical School, University of Birmingham, Edgbaston, Birmingham B15 2TT, UK

3 Department of Urogynaecology and Pelvic Floor Medicine, Birmingham Women's Hospital, Birmingham, UK case. While one possible explanation for under-presenting may relate to the stigma attached to incontinence, it is conceivable that other factors, such as the patient's psychological traits and states, may also play a part.

Current diagnostic and treatment guidelines [6] include a range of conservative, pharmacological and surgical interventions [7]. However, many of these recommendations are not based on high-quality evidence from randomised controlled trials, but rather on the opinions and experience of the Guideline Development Group [7]. It is worth noting that there is a considerable body of anecdotal and research evidence that suggests successful clinical treatment of UI does not necessarily correlate with patients' personal evaluations of outcome, quality of life or general satisfaction [8]. Given this, and the fact that psychological stress and distress may be implicated in UI, it would seem logical to suppose that individual predisposition, personality characteristics, traits and states could potentially play a role in the management of UI. If this contention carries any weight, then it would seem reasonable to consider the psychological status of women presenting with UI, on the basis that any factor adversely affecting recovery and outcomes might be managed alongside the clinical problems.

This very brief overview highlights three points that are germane to the current paper:

1. The low presentation rates of women with UI compared to population estimates of the condition might be in part a product of the stigma associated with incontinence, but it is also possible that individual differences may also influence whether or not a woman presents for treatment.

2. The role of psychological factors in UI, its nature, severity and outcomes, suggests that managing the clinical presentation of UI without simultaneously addressing the psychological correlates may be of less value than managing the patient as a whole. 
3. There are a surprisingly high number of treatment guidelines contained in the National Institute for Health and Care Excellence (NICE) protocols that are based on clinical judgement, suggesting that factors other than those that are objectively quantifiable may be relevant to intervention decisions. In short, core personality characteristics might be a central factor in all aspects of UI, from initial presentation through to assessment of treatment outcomes.

There is extensive evidence that personality may be a significant correlate of: health and well-being, vulnerability to disease, recovery from illness and the ability to cope with chronic conditions [9]. Most clinical guidelines recommend that interventions are patient-centred, implying that individual differences may be important in coping, compliance and treatment outcomes [6].

\section{Components of hardiness}

Relevant to this line of reasoning is the trait of hardiness. It is defined as a coping style which has the capacity to protect the individual against the adverse consequences of stress [10] and comprises three core components: control (personal feelings of mastery over life events), challenge (the predisposition to regard difficult situations as a challenge rather than a threat) and commitment (the ability to engage with daily life in a positive and constructive way). All three components involve behavioural, cognitive and affective approaches to coping.

\section{Hardiness: an important correlate for health}

Research conducted to date on the relationship between hardiness and health has been promising. As a dispositional trait, there is evidence that it is a better predictor of a range of health outcomes than other personal characteristics, such as locus of control, optimism or self-esteem [11], and indeed several research studies demonstrate a convincing link between levels of hardiness and health status. For example, high levels of hardiness have been shown to be positively correlated with better outcomes for patients with chronic illnesses [12], with lower levels of depression and fatigue in caregivers [13] and with recovery from traumatic events [14]. Similarly, hardiness has also been shown to be a factor in preventive health, with individuals high in hardiness being more likely to pursue positive health practices and to pursue effective health strategies [15]. A synthesis of the available evidence of the relationship between hardiness and chronic illness suggests that hardiness provides the individual with the capacity to adapt constructively and to shore up coping processes [12].

Nonetheless, its promotion as a characteristic in a patient population may be counterproductive, since while it may enable individuals to withstand the effects of stress, it may also encourage them to ignore symptoms [16].

\section{Hardiness and UI}

However, the balance of evidence tends to suggest that hardiness, through its enhanced sense of control, engagement with a problem and the reconstruction of the significance of an adverse event, may mediate the effective management of chronic conditions. It is surprising, therefore, that there is a paucity of hardiness research in the area of female UI, especially given the established interrelationship between psychological state and incontinence [17]. While the association is complex, it would seem that psychological morbidities may be implicated in both the cause of UI as well as its impactparticularly the woman's capacity to cope [18]. On this basis, then, it could be argued that if negative psychological states, such as depression and subjective distress, are associated with negative reactions to UI, then it might also be the case that the trait of hardiness might be related to its constructive management, both pre- and post-diagnosis. From this, a number of hypotheses emerge. Because of the disposition of hardy individuals to cope constructively with adverse events, it could be reasonably hypothesised that women who are high in hardiness might:

- Be slower to present with symptoms of UI (especially where these are mild or moderate)

- Be less likely to report poor UI-related quality of life and subjective distress

- Be more compliant with clinical interventions

- Be more satisfied with treatment

- Be more resilient in the event of subsequent problems

Conversely, women low in hardiness would show the reverse.

If these preliminary hypotheses have any substance, they have clear indications for clinical practice. Firstly, late presentation with UI may compromise effective clinical treatment, and therefore it would seem imperative to capture the problem in its early stages. Conversely, in early presentation, where minor symptoms or the thought of symptoms (which may be as debilitating and tie into catastrophic behaviour) have a huge impact, improvements may be more difficult to achieve. In this sense, the predisposition of the hardy individual to cope with adversity by pursuing their own autonomy may militate against early diagnosis of UI and therefore against successful treatment.

There is also a possibility that hardy women may be more inclined to seek medical help once they have recognised that their own resources for coping with UI are insufficient. On the other hand, it could be hypothesised that low hardy women with UI are less likely to present for treatment, as according to some authors [10, 24] low hardiness is clinically linked to avoidance of coping mechanisms. 
To establish whether or not severity of symptoms/late presentation of UI is linked to high hardiness (and early presentation and fear of the impact of symptoms is associated with low hardiness) scores, a measure of this characteristic could be routinely used at the point of referral. With regard to the second hypothesis, it might be expected that women presenting to the specialist UI clinic may report differential levels of distress and quality of life relative to their hardiness ratings. Given that the hardy individual may re-construe the symptoms of UI as a challenge to be overcome, it is conceivable that they may also underestimate the severity and significance of the symptoms and their associated problems, while having higher quality of life appraisals than might be expected from the clinical presentation. Conversely, low-hardiness women might overstate symptoms and their impact. Either way, realistic evaluation of the problem may be of value in managing the reality of UI. Failure to recognise this as a factor may, for example, result in a woman who has a "good outcome" clinically in symptom resolution, but the fear of failure, or failure to restore confidence, means that behaviour patterns remain and there is no improvement in reported quality of life despite symptom moderation/cure.

The third hypothesis suggests that women high in hardiness, because of their high sense of commitment and control, may be more likely to comply with clinical intervention, such as pelvic floor exercises, while women low on this trait may display less compliance. Given the importance of selfmanagement through, for example, diet and exercise [19] as stand-alone or adjuvant therapy for UI, those individual differences that promote greater compliance might be worthy of investigation and development. Here, the associated dynamic state of resilience, with its potential for change and modification, may be relevant in the management of UI.

Some authors suggest that resilient/hardy women can implement necessary lifestyle modifications in order to avoid UI [20]. While the trait of hardiness is unlikely to respond to significant modification, since it is inherently stable, the associated transitory state of resilience offers more opportunity for change, and there are several examples of such training programmes [21-23]. In this context, teaching patients coping strategies to manage their UI may help to decrease the severity of the condition or avoid its development.

Holding objective clinical measures constant, it would be anticipated that patient satisfaction with treatment and quality of life scores would be greater in women recording higher levels of hardiness, on the grounds that the three core factors subsumed under hardiness would render them more positive about their experiences and current health/UI status, irrespective of clinical condition. If this hypothesis was supported, then it would go some way towards explaining the documented variations in personal perceptions of treatment outcomes relative to actual clinical outcome. Finally, these components of hardiness may render women high on hardiness to be able to withstand any shortcomings in treatment and subsequent problems associated with UI.

\section{The hardiness scales}

Underpinning these predictions are a number of methodological issues that would need to be resolved before any research study could be initiated, of which a key one is the selection of an appropriate hardiness scale that would be valid, reliable and relevant to the population under consideration. Screening for hardiness can improve clinical care in various contexts [24]. While a number of such scales are available (e.g. The Dispositional Resilience Scale, Bartone et al., 1989; The Mental Toughness 48 Questionnaire, Clough et al., 2002; The Resilience Scale, Wagnild and Young, 1993) [25], they vary considerably in their psychometric properties, ease of use, development credentials and relevance for specific samples. For any sort of hardiness assessment to be routinely carried out either in primary care or in specialist urogynaecology clinics, it would be essential for the instrument to have demonstrable application and sample relevance, to be easy to use and interpret and to have the sensitivity and the discriminant and predictive capacity to be able to identify those women whose levels of resilience may impede the management of their UI. Once that had been established, then the selected scale could be routinely used on all consenting women presenting with UI and reissued at various stages in their treatment to investigate whether or not the theoretical links between hardiness and UI highlighted in the hypotheses exist. If any of the hypotheses were found to be supported, then the findings would make a case for using psychological programmes to develop those characteristics that predisposed women to respond optimally to interventions.

The above case for routinely assessing and using personal resilience as a means of enhancing the management of UI is, as yet, just speculation, but the argument for investigating its possible role in the evolution and management of UI is a compelling one. Undoubtedly, there is a wealth of incontrovertible evidence that attests to the range of personal characteristics that play a part in health and illness; it is entirely plausible, therefore, that hardiness, with its ability to mediate stress and distress, should be a relevant factor in a condition whose scope, severity and successful treatment are so heavily affected by these characteristics.

\section{Conflicts of interest None}

Authors' contributions FIB: literature search, manuscript writing and editing. SL and LS: manuscript writing and editing. PTH: conceived the idea, manuscript writing and editing.

\section{References}

1. Hunskaar S, Lose G, Sykes D, Voss S (2004) The prevalence of urinary incontinence in women in four European countries. BJU Int 93(3):324-330 
2. Shaw C, Gupta RD, Bushnell DM, Assassa RP, Abrams P, Wagg A, Mayne C, Hardwick C, Martin M (2006) The extent and severity of urinary incontinence amongst women in UK GP waiting rooms. Fam Pract 23(5):497-506

3. The Continence Foundation (2001) http://www.continencefoundation.org.uk/. Accessed 18 Jul 2014

4. Shaban A, Drake MJ, Hashim H (2010) The medical management of urinary incontinence. Auton Neurosci 152(1-2):4-10

5. Riss P, Kargl J (2011) Quality of life and urinary incontinence in women. Maturitas 68(2):137-142

6. NICE (2013) Urinary incontinence: the management of urinary incontinence in women. http://www.nice.org.uk/Guidance/CG171. Accessed 18 Jul 2014

7. Smith A (2013) Management of urinary incontinence in women: a summary of updated NICE guidelines. BMJ; http://www.bmj.com/ content/347/bmj.f5170. Accessed 18 Jul 2014

8. Loane KH (2010) The psychological aspects of women suffering urinary incontinence and their experience of treatment. Unpublished MD thesis, University of Birmingham

9. Smith TW, MacKenzie J (2006) Personality and risk of physical illness. Annu Rev Clin Psychol 2:435-467

10. Kobasa SC, Maddi SR, Kahn S (1982) Hardiness and health: a prospective study. J Pers Soc Psychol 42:168-177

11. Eschelman KJ, Bowling NA, Alarcon GM (2010) A meta-analytic examination of hardiness. Int J Stress Manage 17(4):277-307

12. Brooks MV (2003) Health-related hardiness and chronic illness: a synthesis of current research. Nurs Forum 38(3):11-20

13. Clark PC (2002) Effects of individual and family hardiness on caregiver depression and fatigue. Res Nurs Health 25(1):37-48

14. Taylor MK, Pietrobon R, Taverniers J, Leon MR, Fern BJ (2013) Relationships of hardiness to physical and mental health status in military men: a test of mediated effects. J Behav Med 36(1):1-9
15. Weibe DJ, McCallum DM (1986) Health practices and hardiness as mediators in the stress-illness relationship. Health Psychol 5:425438

16. Lee HJ (1983) Analysis of a concept: hardiness. Oncol Nurs Forum 10(4):32-35

17. Yip SK, Cardozo L (2007) Psychological morbidity and female urinary incontinence. Best Pract Res Clin Obstet Gynaecol 21(2): 321-329

18. Sievert KD, Amend B, Toomey PA et al (2012) Can we prevent incontinence? ICI-RS 2011. Neurourol Urodyn 31(3):390-399

19. Wing RR, Creasman MS, Smith West D et al (2010) Program to reduce incontinence by diet and exercise: improving urinary incontinence in overweight and obese women through modest weight loss. Obstet Gynecol 116(2 Pt 1):284-292

20. Jamison M, Weidner A, Romero A, Amundsen C (2007) Lack of psychological resilience: an important correlate for urinary incontinence. Int Urogynecol J Pelvic Floor Dysfunct 18:1127-1132

21. Judkins S, Reid B, Furlow L (2006) Hardiness training among nurse managers: building a healthy workplace. J Contin Educ Nurs 37:202-207

22. Bartone P (2012) Social and organizational influences on psychological hardiness: how leaders can increase stress resilience. Secur Inform 1(1):1-10

23. Bartone P (2006) Resilience under military operational stress: can leaders influence hardiness? Mil Psychol 18(1):131-148

24. Bartone P, Hystad SW, Eid J et al (2012) Psychological hardiness and coping style a risk/resilience factors for alcohol abuse. Mil Med 177(5):517-524

25. Smith-Osborne A, Whitehill Bolton K (2013) Assessing resilience: a review of measurements across the life course. J Evid Based Soc Work 10(2):111-12616 